\title{
Incidence and survival outcomes of early male breast cancer: a population-based comparison with early female breast cancer
}

\author{
Yan Wang ${ }^{1,2 \#}$, Kai Chen ${ }^{1,2 \#}$, Yaping Yang ${ }^{1,2 \#}$, Luyuan Tan ${ }^{1,2}$, Lili Chen ${ }^{1,2}$, Liling Zhu ${ }^{1,2}$, Fengxi Su ${ }^{1,2}$, \\ Xue Liu ${ }^{3}$, Shunrong $\mathrm{Li}^{1,2}$ \\ ${ }^{1}$ Guangdong Provincial Key Laboratory of Malignant Tumor Epigenetics and Gene Regulation, Sun Yat-sen Memorial Hospital, Sun Yat- \\ sen University, Guangzhou 510120, China; ${ }^{2}$ Department of Breast Surgery, Breast Tumor Center, Sun Yat-sen Memorial Hospital, Sun Yat-sen \\ University, Guangzhou 510120, China; ${ }^{3}$ College of Basic Medicine, Jining Medical University, Jining 272067, China \\ Contributions: (I) Conception and design: S Li, X Liu, F Su, Y Wang, K Chen; (II) Administrative support: S Li, K Chen, F Su; (III) Provision of \\ study materials or patients: Y Wang, K Chen, Y Yang; (IV) Collection and assembly of data: Y Wang, L Tan, L Chen, L Zhu; (V) Data analysis and \\ interpretation: Y Wang, K Chen, Y Yang; (VI) Manuscript writing: All authors; (VII) Final approval of manuscript: All authors. \\ \#The authors contributed equally to this work. \\ Correspondence to: Shunrong Li, Fengxi Su. Department of Breast Surgery, Breast Tumor Center, Sun Yat-sen Memorial Hospital, Sun Yat-sen \\ University, 107 Yanjiang West Road, Guangzhou 510120, China. Email: lishr@mail.sysu.edu.cn; sufengxi@mail.sysu.edu.cn. Xue Liu, College of \\ Basic Medicine, Jining Medical University, 16 Hehua Road, Jining 272067, China. Email: xuelingjing@yeah.net.
}

Background: Male breast cancer (MBC) is a rare malignancy. We aimed to analyze the incidence trends, clinicopathological characteristics, and survival outcomes in early MBC comparison with early female breast cancer (FBC).

Methods: We included eligible MBC and FBC patients with stage I-II disease in the Surveillance, Epidemiology, and End Results (SEER) database from 2000-2015. Joinpoint regression was used to evaluate the trends in age-adjusted incidence. A one-to-four propensity score matching (PSM) analysis was performed to reduce bias in a retrospective study. Survival outcomes were evaluated using Kaplan-Meier analyses with the log-rank test and Cox proportional hazards regression analysis.

Results: Trends in the age-adjusted incidence rates of early MBC were stable [2000-2015, annual percentage change $(\mathrm{APC})=0.50,95 \%$ confidence interval (CI): -0.1 to $1.1, \mathrm{P}=0.102]$; however, the incidence of early FBC changed significantly over the time period (2000-2015, APC $=0.30,95 \%$ CI: 0.0 to 0.6 , $\mathrm{P}=0.045)$. In the matched cohort, unmarried status, higher grade, larger tumor size, and advanced lymph node (LN) status were associated with a higher risk of breast cancer death and death of any causes both in early MBC and FBC patients. The hormone receptor (HR) status was as a prognostic factor in FBC patients, but not in MBC. Early MBC had worse breast cancer-specific survival (BCSS) and overall survival (OS) than early FBC in stage I, stage II and HR-positive subgroup of patients.

Conclusions: The biological behavior, clinicopathological features, and clinical outcomes of early MBC are different from that of FBC. Further studies on individualized treatment approaches in MBC are needed.

Keywords: Male breast cancer (MBC); incidence; prognosis; propensity score matching (PSM)

Submitted Sep 24, 2019. Accepted for publication Sep 29, 2019.

doi: 10.21037/atm.2019.10.04

View this article at: http://dx.doi.org/10.21037/atm.2019.10.04

\section{Introduction}

Male breast cancer (MBC) represents an uncommon disease, with an estimated 2,550 men diagnosed in the
United States (USA) in 2018, accounting for less than 1\% of all new breast cancer patients (1). In recent years, the trends in incidence of female breast cancer (FBC) has risen due 
to the increased longevity, changes in lifestyle behaviors, the prevalence of mammography screening and improve healthcare awareness (2-4), and a growing proportion of breast carcinomas are detected early. Unlike FBC, men are not only lack of self-awareness of breast health examination, but also not included in the screening program. Thus, MBCs are tended to advanced stage.

Due to the rarity of $\mathrm{MBC}$, large-scale randomized clinical trials for $\mathrm{MBC}$ are not feasible. Therapeutic strategies in men are, therefore, the generalized from the management of FBC patients. The optimal management strategy for MBC patients is still unknown. MBC patients have significantly different clinicopathological features and treatments when compared to that of FBC patients. For example, male patients had different hormone levels at baseline and were diagnosed at an older age and more advanced stage $(5,6)$. Furthermore, MBC patients are more likely to undergo total mastectomy $(5,7)$. To address the issues mentioned above, a study using the real-world data from national cancer-registry database would be informative and clinically relevant. The Surveillance, Epidemiology, and End Results (SEER) database is a national database that covered approximately $28 \%$ of the USA population. In this study, we aimed to use the SEER database to analyze the trends in incidence, clinicopathological characteristics, and survival outcomes of early MBC patients and compared them with the propensity score-matched early FBC population.

\section{Methods}

\section{Study population}

We retrospectively identified male and female patients with stage I-II breast cancer between 2000 and 2015 from the National Cancer Institute's SEER 18 registries. The exclusion criteria for the analysis of survival outcomes were as follows: (I) patients who were younger than 18 years old at diagnosis; (II) patients who had another primary malignancy before the diagnosis of breast cancer; (III) patients who had unknown information on race, marital status, estrogen receptor (ER) status, progesterone receptor (PR) status, surgical procedure and survival; (IV) patients who diagnosed with inflammatory breast cancer or phyllode tumors; (V) patients with only death certification and autopsy data.

Clinicopathological factors including age at diagnosis, year of diagnosis, race, marital status, histology, grade,
American Joint Committee on Cancer (AJCC) stage, tumor size, LN status, ER status, PR status, human epidermal growth factor receptor 2 (Her2) status, molecular subtype, surgery, chemotherapy and radiation were retrieved from the SEER database. Hormone receptor (HR) positivity was defined as ER and/or PR positivity. Her2 status and subtype were not available in the SEER database until 2010. Because the HR-negative/Her2-positive subtype of MBC was uncommon (8), breast cancer molecular subtypes were classified as HR-positive/Her2-negative (ER/PR+ and Her2-), Her2-positive (Her2+, regardless of ER/PR status) and triple-negative (ER-, PR- and Her2-). This is a study using de-identified, public available dataset, and ethical approval can be waived based on our institutional policy.

\section{Statistical analysis}

The incidence rates per year of stage I-II breast cancer in the male and female cohorts are calculated by SEER*Stat (Version 8.3.5). Trends in the incidence of early MBC and FBC were calculated per 100,000 individuals and were ageadjusted to the USA standard population in the year 2000. The joinpoint regression model is useful for distinguishing and describing the occurrence of changes in different periods throughout trends in data (9). This regression model of cancer incidence rates was used to assess the annual percentage change (APC) in breast cancer. The model allows up to 2 joinpoints for rates from 2000 to 2015 , with 3 maximum segments (10). The incidence rate analyses were performed the Joinpoint statistical software (Version 4.7.0.0) from the Surveillance Research Program of the USA National Cancer Institute.

A chi-square test was used to analyze the differences in demographics, clinicopathological characteristics, and treatments between two groups. Breast cancer-specific survival (BCSS) was measured from the date of diagnosis of breast cancer to the date of death from breast cancer. Overall survival (OS) was measured from the date of diagnosis of breast cancer to the date of death from any cause or the last follow-up. BCSS and OS were assessed using Kaplan-Meier survival methods, and the two cohorts were compared using a log-rank test. Univariate and multivariate Cox regression was used to assess the prognostic factors associated with BCSS and OS.

Eligible patients were considered as the original cohort, which is used to analyze the baseline clinicopathological features and survival of $\mathrm{MBC}$ and $\mathrm{FBC}$ in a real-world scenario. To reduce bias, we developed a 1:4 (MBC:FBC) 

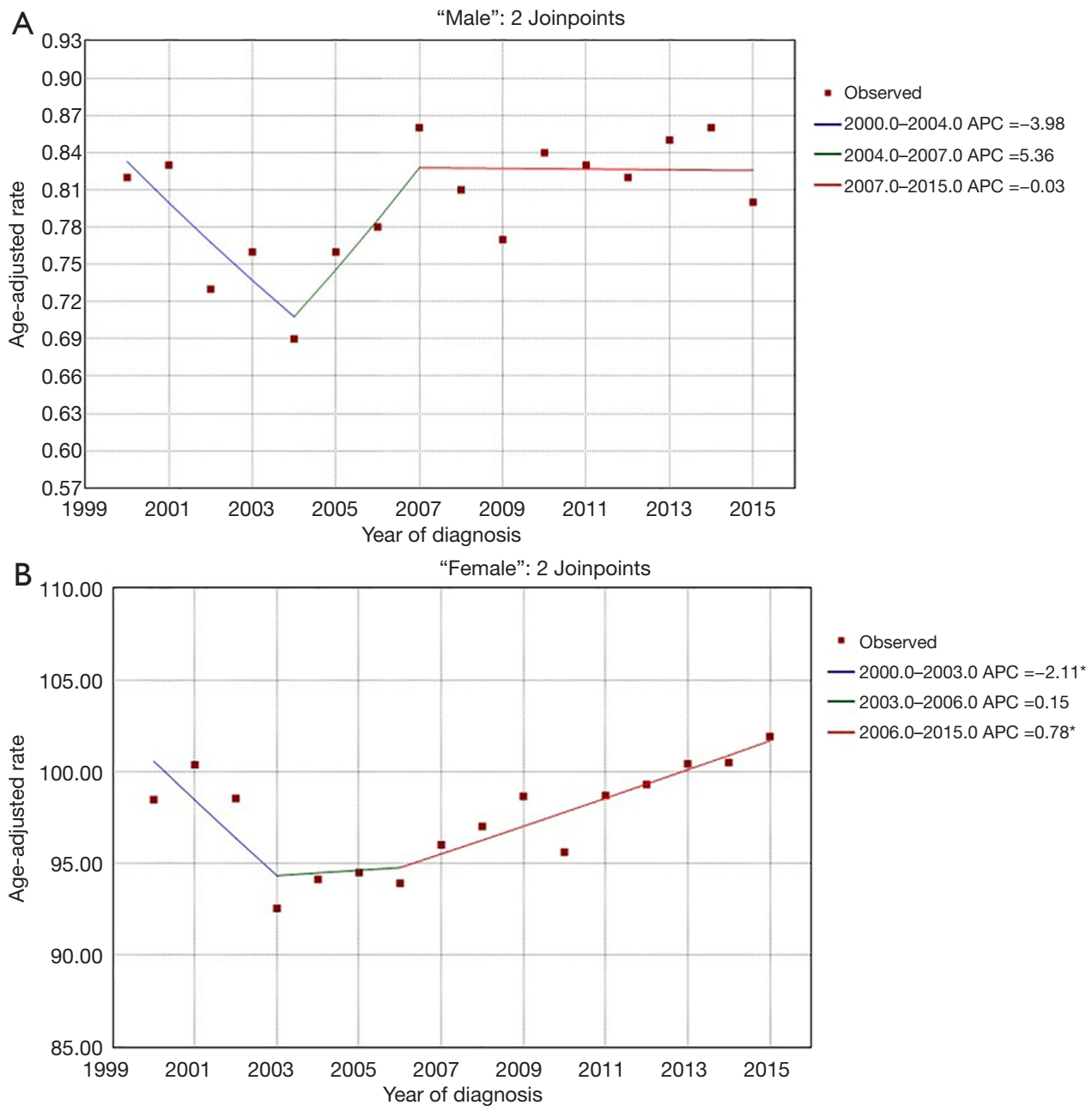

Figure 1 Trends in the age-adjusted incidence rates of early male breast cancer (MBC) (A) and female breast cancer (FBC) (B) by joinpoint regression (2000-2015). *, indicates that the annual percent change (APC) is significantly different from zero at the alpha $=0.05$ level. Final Selected Model: 0 Joinpoint.

matched cohort using PSM analysis. All statistical tests were two-sided, $\mathrm{P}$ values of 0.05 or less were considered significant. Statistical analysis was performed using SPSS software, version 23 (IBM Corporation, Armonk, NY, USA).

\section{Results}

\section{Trends in age-adjusted incidence, 2000-2015}

During the period from 2000 to 2015, the age-adjusted incidence rates of early $\mathrm{MBC}$ and $\mathrm{FBC}$ are listed in Figure $1 A, B$, respectively. Trends in the incidence rates of early MBC in USA were stable [2000-2015, APC
$=0.50,95 \%$ confidence interval $(\mathrm{CI}):-0.1$ to $1.1, \mathrm{P}=0.102$; 2000-2004, APC $=-3.98,95 \%$ CI: -8.0 to $0.2, \mathrm{P}=0.060$; 2004-2007, $\mathrm{APC}=5.36,95 \% \mathrm{CI}:-8.2$ to $21.0, \mathrm{P}=0.409$; 2007-2015, APC $=-0.03,95 \% \mathrm{CI}:-1.4$ to $1.3, \mathrm{P}=0.956$ ] (Figure 1A). However, the age-adjusted incidence rates of early $\mathrm{FBC}$ had changed significantly over the time period (2000-2015, APC $=0.30,95 \%$ CI: 0.0 to $0.6, \mathrm{P}=0.045$ ) (Figure $1 B$ ). The results indicated that incidence rates for FBC decreased each year during 2001 to 2003, with a sharp decrease (5.98\%) occurring from 2002 to 2003 (2000-2003, $\mathrm{APC}=-2.11,95 \% \mathrm{CI}:-4.1$ to $-0.1, \mathrm{P}=0.040)$. Early FBC incidence rates did not change significantly from 2003 to $2006(2003-2006$, APC $=0.15,95 \%$ CI: -3.8 to 4.2 , $\mathrm{P}=0.931$ ). However, the incidence rates for $\mathrm{FBC}$ increased 
Table 1 Patient characteristics before and after propensity score matching

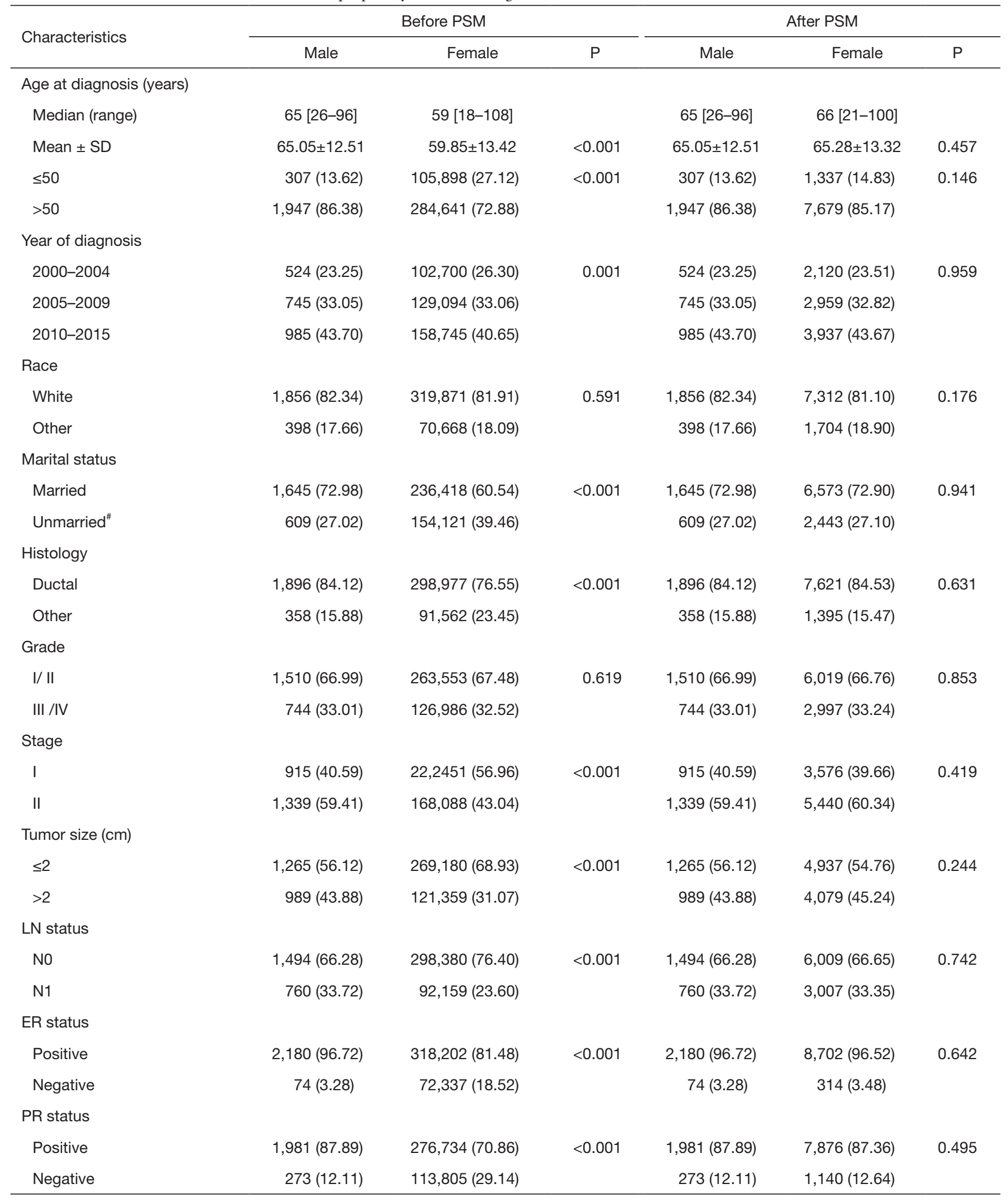

Table 1 (continued) 
Table 1 (continued)

\begin{tabular}{|c|c|c|c|c|c|c|}
\hline Characteristics & \multicolumn{3}{|c|}{ Before PSM } & \multicolumn{3}{|c|}{ After PSM } \\
\hline \multicolumn{7}{|l|}{ HR status } \\
\hline Positive & $2,197(97.47)$ & 322,853 (82.67) & $<0.001$ & 2,197 (97.47) & 8,753 (97.08) & 0.321 \\
\hline Negative & $57(2.53)$ & $67,686(17.33)$ & & $57(2.53)$ & $263(2.92)$ & \\
\hline \multicolumn{7}{|l|}{ Her2 status* } \\
\hline Positive & $104(10.56)$ & 21,877 (13.78) & 0.003 & $104(10.56)$ & $449(11.40)$ & 0.456 \\
\hline Negative & $881(89.44)$ & 136,868 (86.22) & & $881(89.44)$ & $3,491(88.60)$ & \\
\hline \multicolumn{7}{|l|}{ Molecular subtype* } \\
\hline $\mathrm{HR}+/$ Her2- ${ }^{\mathrm{a}}$ & $870(88.32 \%)$ & $119,552(75.31)$ & $<0.001$ & 870 (88.32) & $3,431(87.08)$ & 0.465 \\
\hline BCS & $257(11.40)$ & 249,923 (63.99) & $<0.001$ & $257(11.40)$ & $1,074(11.91)$ & 0.502 \\
\hline Mastectomy & $1,997(88.60)$ & $140,616(36.01)$ & & $1,997(88.60)$ & 7,942 (88.09) & \\
\hline \multicolumn{7}{|l|}{ Chemotherapy } \\
\hline Yes & 799 (35.45) & 152,109 (38.95) & 0.001 & 799 (35.45) & $3,184(35.31)$ & 0.906 \\
\hline No & $1,455(64.55)$ & $238,430(61.05)$ & & $1,455(64.55)$ & $5,832(64.69)$ & \\
\hline \multicolumn{7}{|l|}{ Radiation } \\
\hline Yes & $478(21.21)$ & $213,758(54.73)$ & $<0.001$ & $478(21.21)$ & 1,748 (19.39) & 0.052 \\
\hline No & $1,776(78.79)$ & $176,781(45.27)$ & & $1,776(78.79)$ & $7,268(80.61)$ & \\
\hline
\end{tabular}

\#, unmarried status includes divorced, separated, single, and widowed; *, Her2 status and molecular subtype included data from $2010-$ 2015; ' , HR+/Her2-: ER+ or PR+, Her2-; ' , HER2+: Her2+, regardless of ER or PR status; ' , triple-negative: ER-, PR-, Her2-. PSM, propensity score matching; LN, lymph node; ER, estrogen receptor; PR, progesterone receptor; HR, hormone receptor; Her2, human epidermal growth factor receptor 2; BCS, breast-conserving surgery.

slowly but significantly from 93.94 per 100,000 in 2006 to 101.92 in 2015 (2006-2015, APC $=0.78,95 \%$ CI: 0.4 to 1.1 , $\mathrm{P}=0.001)$ (Figure $1 B)$.

\section{Clinical characteristics and survival outcomes of the original cohort}

In the original cohort, a total of 2,254 MBC and 390,539 FBC were eligible before PSM. The baseline characteristics are summarized in Table 1. The median age at diagnosis was 65 (range, 26-96) and 59 (range, 18-108) years for early $\mathrm{MBC}$ and $\mathrm{FBC}$, respectively. Compared with FBC patients, MBCs were more likely to be older at diagnosis $(\mathrm{P}<0.001)$ and married $(\mathrm{P}<0.001)$. Meanwhile, MBC patients had more ductal carcinoma $(\mathrm{P}<0.001)$, advanced-stage disease $(\mathrm{P}<0.001)$, larger tumor size $(\mathrm{P}<0.001)$, positive $\mathrm{LNs}(\mathrm{P}<0.001)$, HR- positive tumor $(\mathrm{P}<0.001)$ and more $\mathrm{HR}+/ \mathrm{Her} 2-$ subtype $(\mathrm{P}<0.001)$. Also, most male patients underwent mastectomy $(\mathrm{P}<0.001)$, but a low percentage of $\mathrm{MBC}$ received chemotherapy $(\mathrm{P}=0.001)$ and radiation $(\mathrm{P}<0.001)$.

The median follow-up period in $\mathrm{MBC}$ and $\mathrm{FBC}$ was 60.5 months [interquartile range (IQR), 31-101 months] and 72 months (IQR, 37-117 months), respectively. During follow-up, 165 patients died of breast cancer, 570 patients died of any cause in the MBC cohort; 20,355 patients died of breast cancer, and 57,351 patients died of any cause in the FBC cohort. Compared to female patients, male patients had significantly worse BCSS $(\mathrm{P}<0.001$, Figure $2 A)$ and OS $(\mathrm{P}<0.001$, Figure $2 B)$ before PSM. The 5- and 10-year BCSS were $94 \%$ and $86 \%$ for $\mathrm{MBC}$, and $96 \%$ and $92 \%$ for $\mathrm{FBC}$, respectively. Also, the 5 - and 10 -year OS rates were $82 \%$ and $61 \%$ for $\mathrm{MBC}$, and $90 \%$ and $79 \%$ for FBC, respectively. 

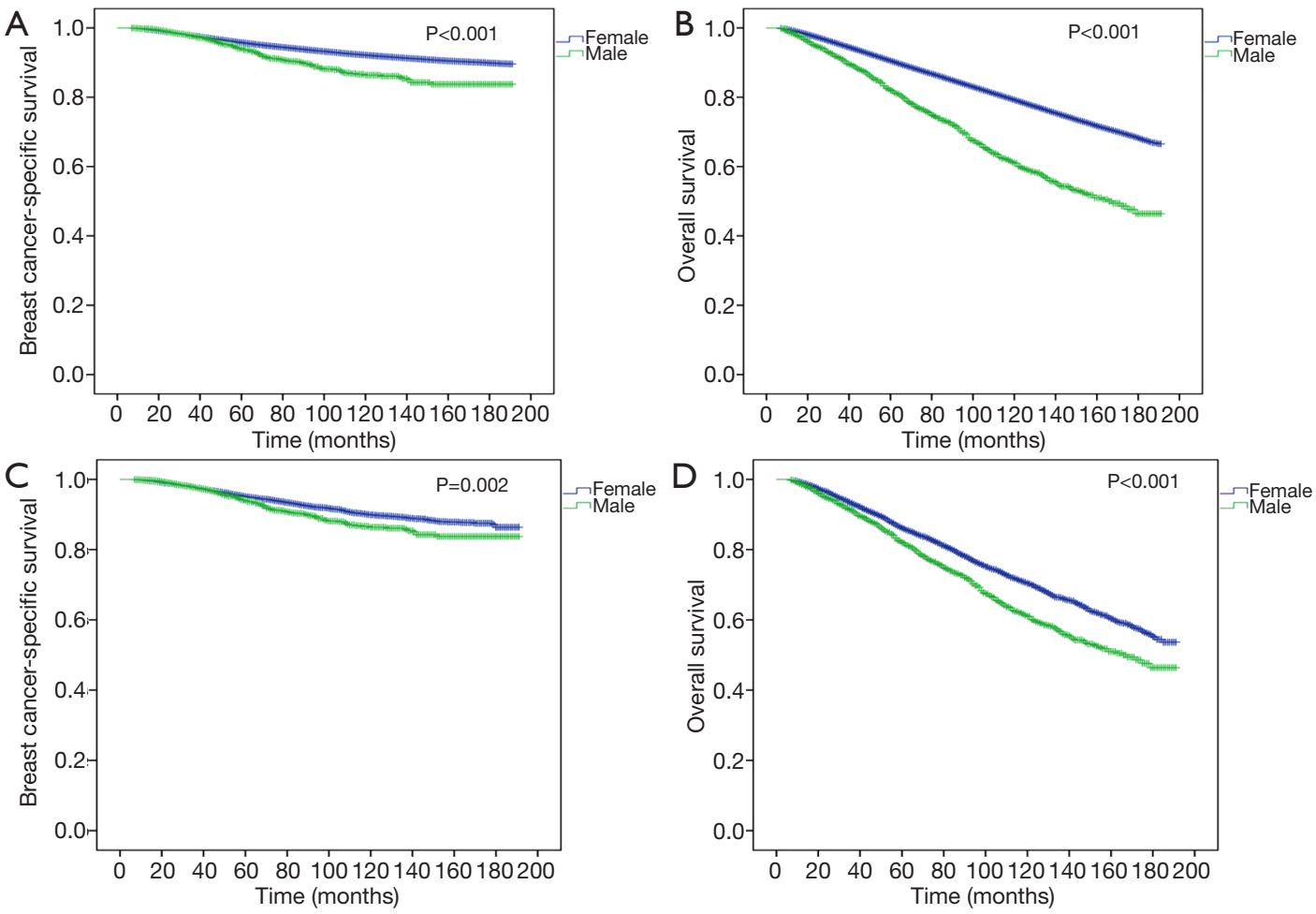

Figure 2 Kaplan-Meier survival curves of breast cancer-specific survival (BCSS) and overall survival (OS) of early male breast cancer (MBC) and female breast cancer (FBC) before (A for BCSS, and B for OS) and after (C for BCSS, and D for OS) propensity score matching (PSM).

\section{Survival outcomes in the matched cohort}

After PSM, 2,254 male and 9,016 female patients were completely matched. None of the variables were significantly different between the two groups (Table 1). In the matched cohort, early MBC had significantly worse BCSS $(\mathrm{P}=0.002$, Figure $2 C)$ and $\mathrm{OS}(\mathrm{P}<0.001$, Figure $2 D)$ than female counterparts.

The results of univariate and multivariate analyses of the prognostic significance in $\mathrm{MBC}$ and $\mathrm{FBC}$ cohorts are listed in Tables 2 and 3, respectively. In male patients, multivariate analysis demonstrated that unmarried status, higher grade, larger tumor size, and advanced LN status were associated with a higher risk of breast cancer death and death of any causes. The histologic type of breast cancer affected BCSS (hazard ratio: 0.612 , 95\% CI: 0.474-0.791, $\mathrm{P}<0.001$ ), but not affect OS. The male patients without chemotherapy had significantly worse OS than those with chemotherapy (hazard ratio: $2.087,95 \%$ CI: $1.710-2.548, \mathrm{P}<0.001$ ). Additionally, HR status did not affect prognosis. In female patients, we observed that patients who were older than 50 years, unmarried status, higher grade, larger tumor size, advanced LN status, HR- negative status and without receiving chemotherapy were associated with a higher risk of breast cancer death and death of any causes. Non-white female patients had better OS than white female patients (hazard ratio: $0.849,95 \%$ CI: $0.746-$ $0.966, \mathrm{P}=0.013)$. The female patients without radiation had significantly worse OS than those with radiation (hazard ratio: $1.262,95 \%$ CI: $1.100-1.448, \mathrm{P}=0.001$ ).

In the stratified analyses, MBC $v s$. FBC was still significantly associated with worse BCSS and OS in patients with stage I and II diseases (Figure 3). The differences were more significant in stage II than in stage I patients. Interestingly, when stratified by HR status, MBC $v s$. FBC was associated with worse BCSS $(\mathrm{P}<0.001$, Figure $4 A)$ and OS $(\mathrm{P}<0.001$, Figure $4 B)$ only in HR-positive subgroups, but not in HR-negative subgroups (Figure $4 C, D$ ).

\section{Discussion}

This large population-based study is the first to describe the trends in incidence of early MBC from 2000 through 2015. There are several differences in biological behaviors and clinicopathological features between male and female 


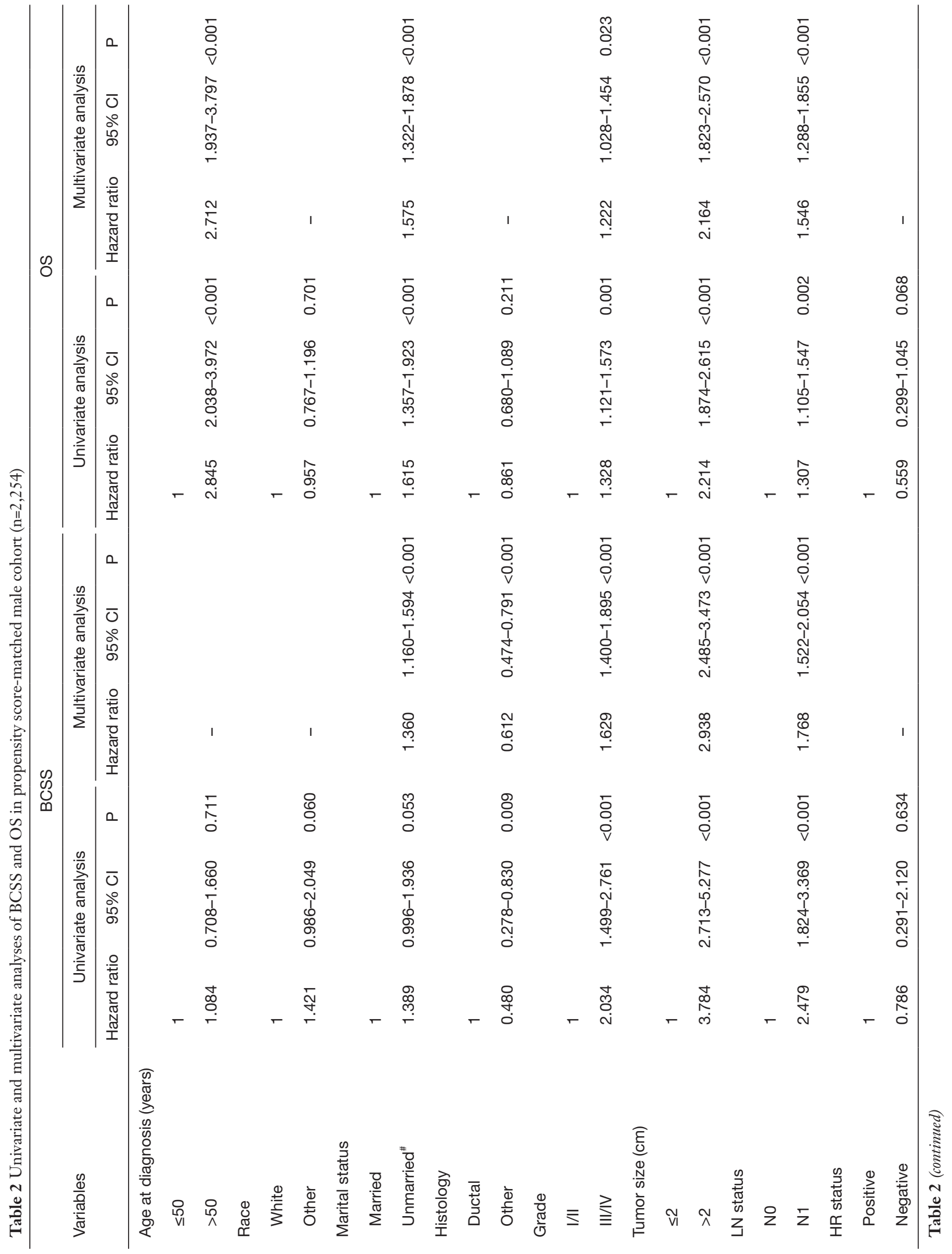




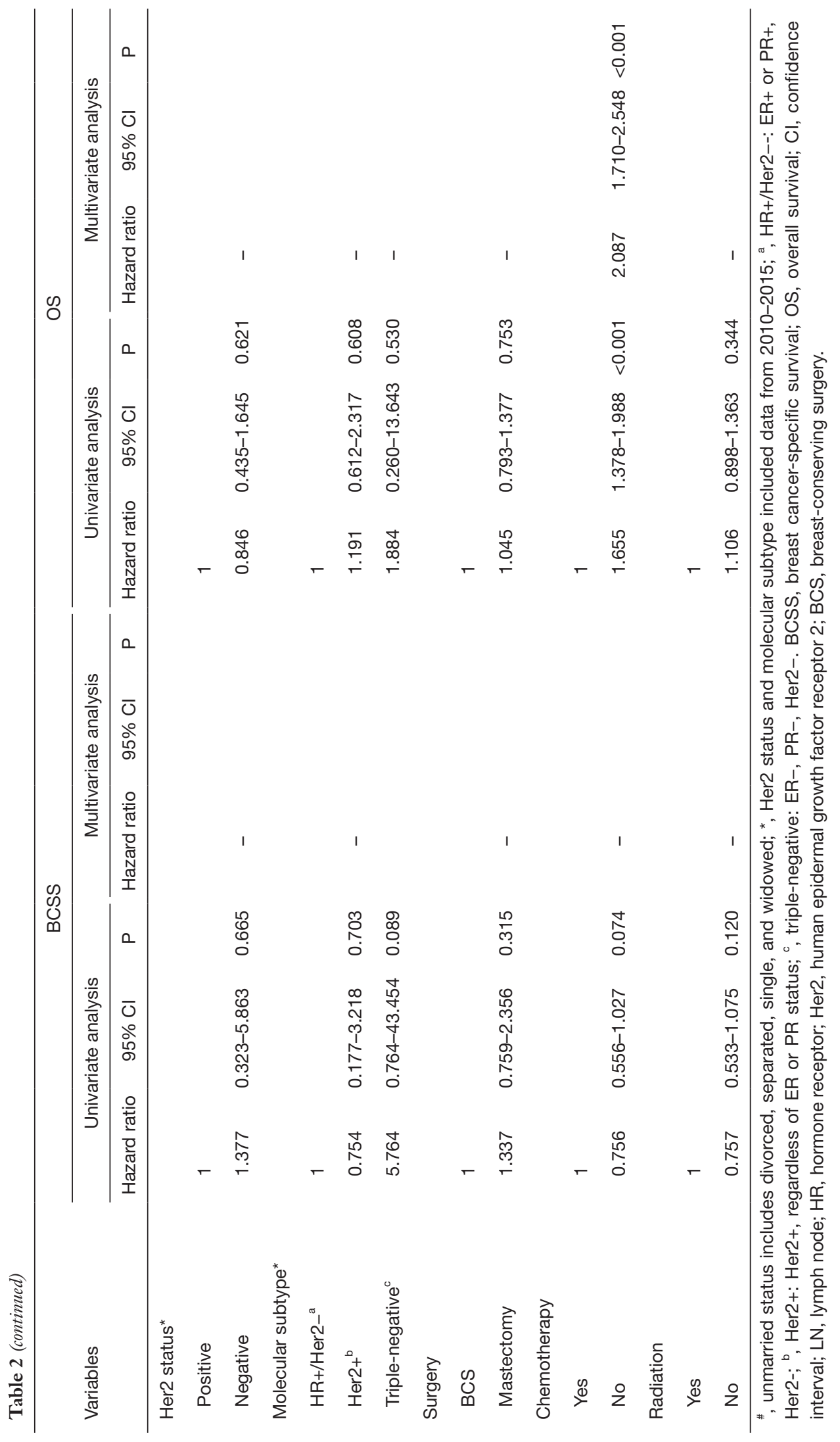




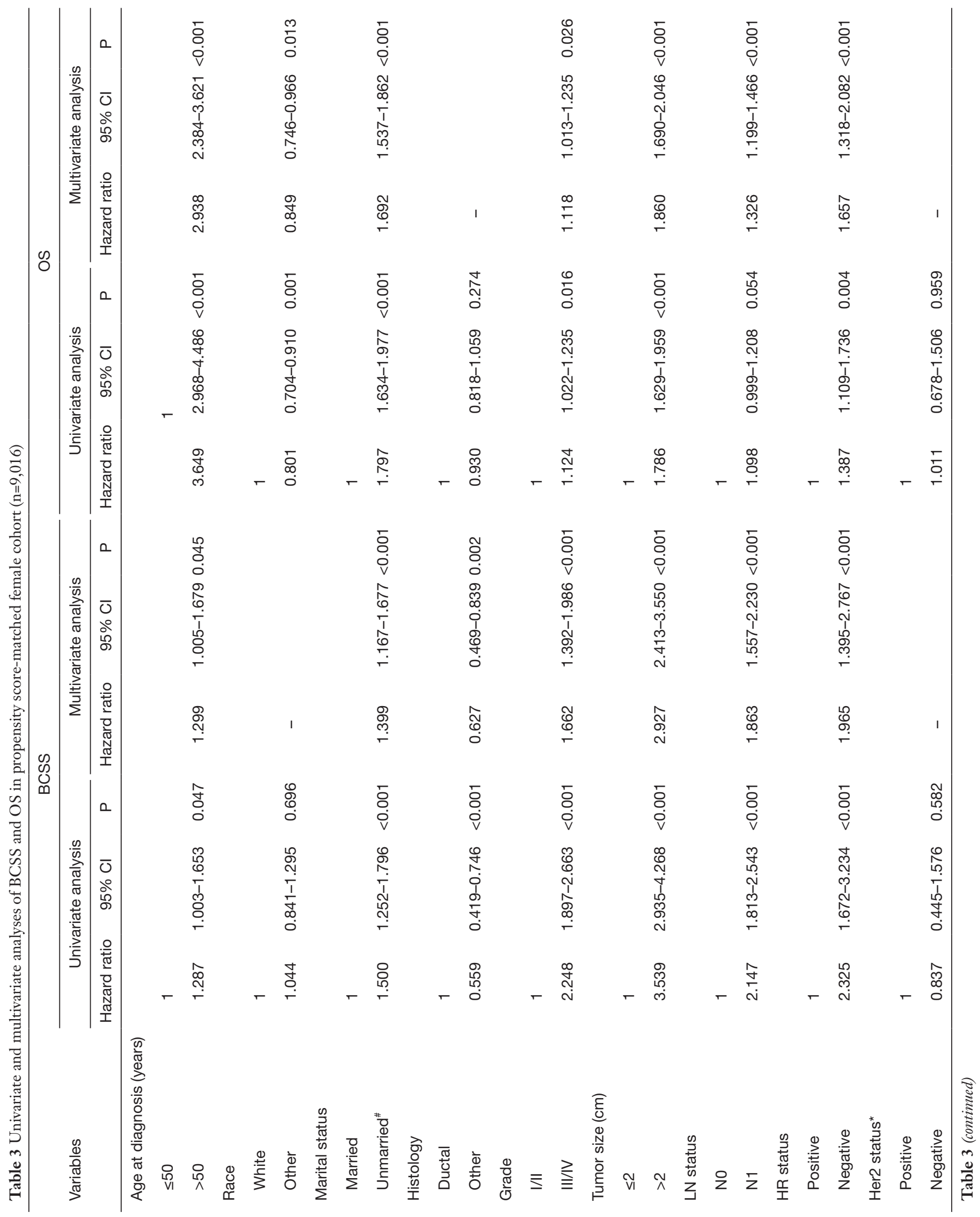




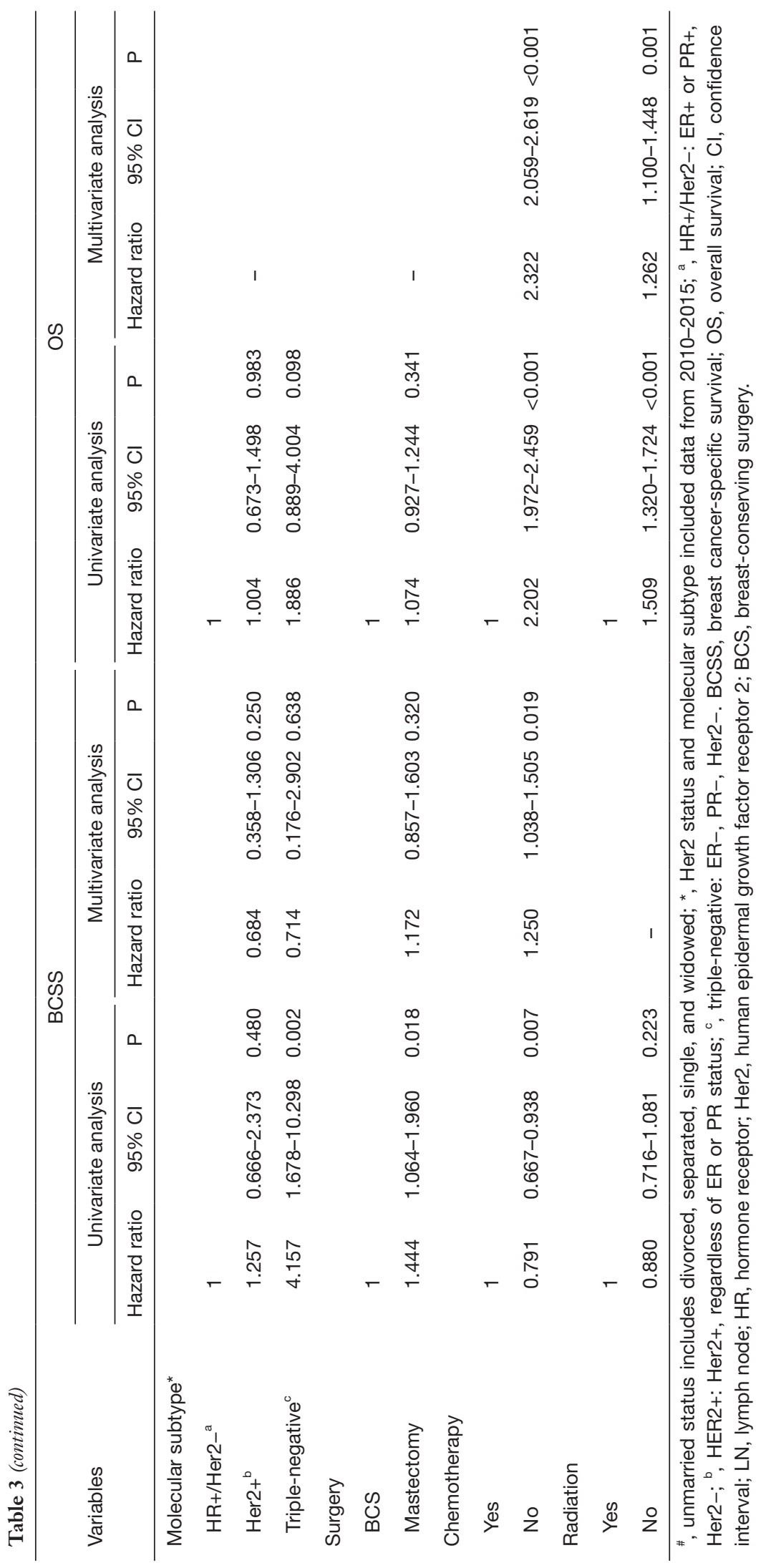



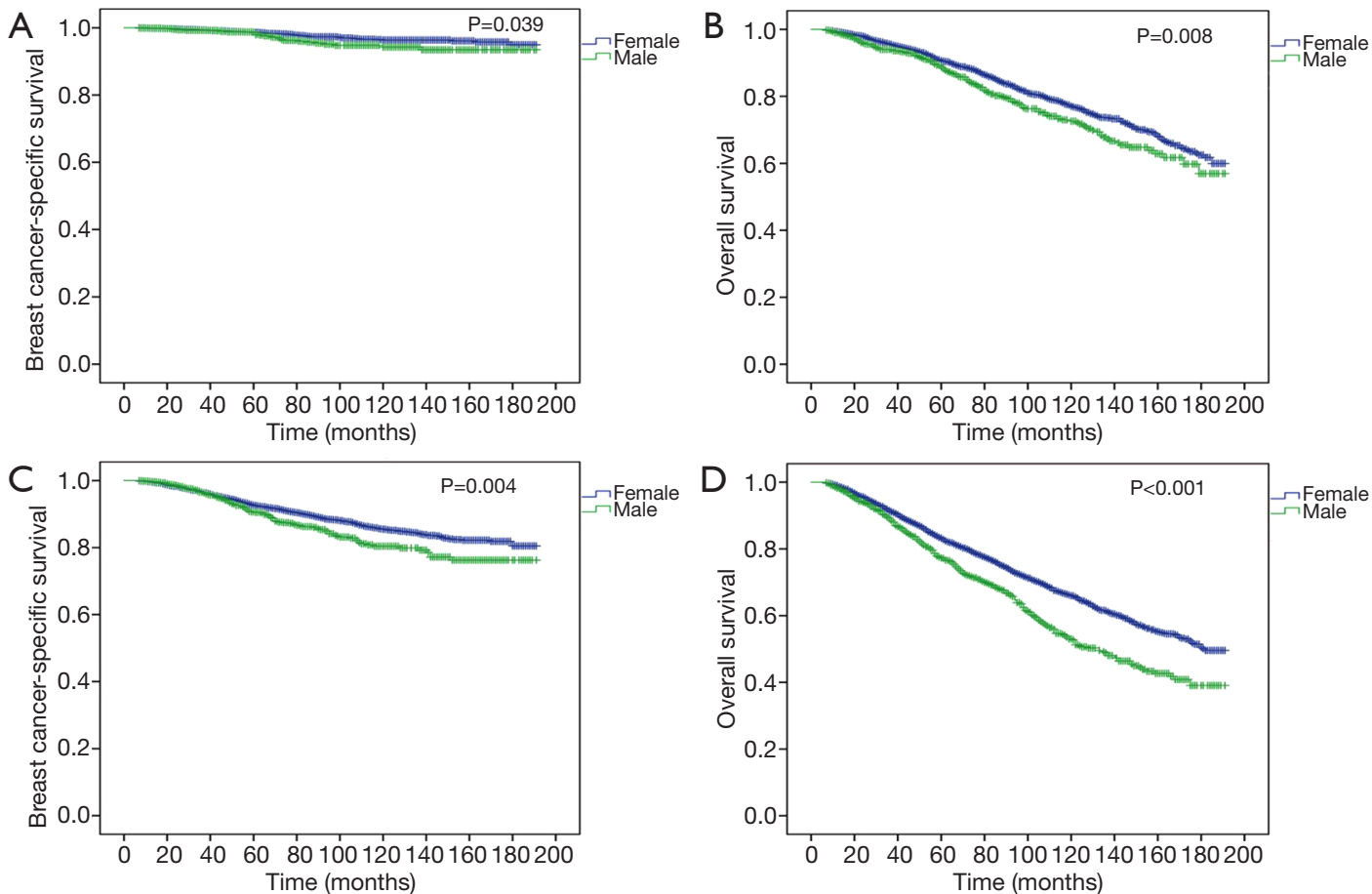

Figure 3 Kaplan-Meier survival curves of breast cancer-specific survival (BCSS) and overall survival (OS) of early male breast cancer (MBC) and female breast cancer (FBC) patients with stage I (A for BCSS, and B for OS) and stage II disease (C for BCSS, and D for OS) after propensity score matching (PSM).
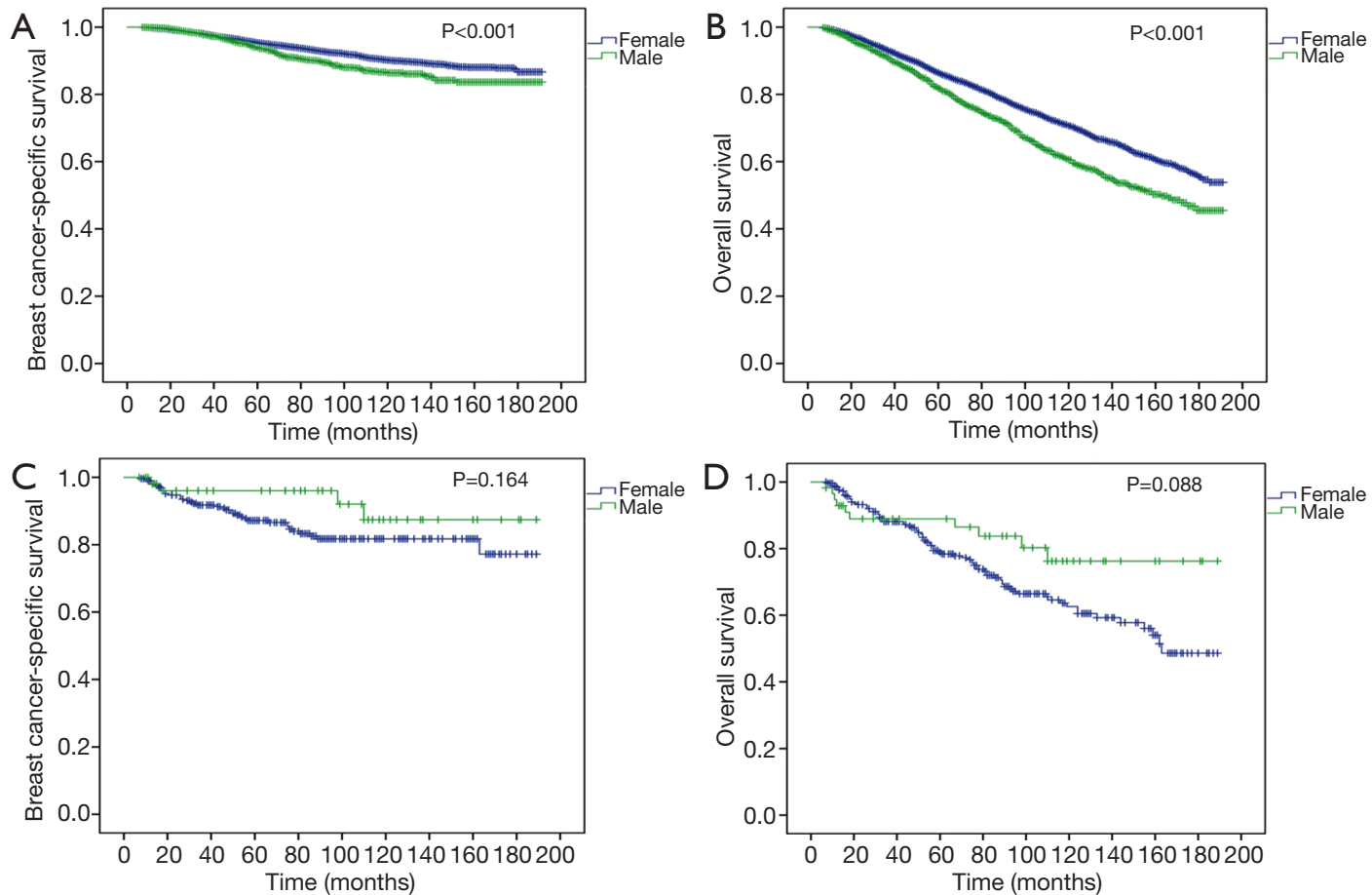

Figure 4 Kaplan-Meier survival curves of breast cancer-specific survival (BCSS) and overall survival (OS) of early male breast cancer (MBC) and female breast cancer (FBC) patients in HR-positive subgroup (A for BCSS, and B for OS) and HR-negative subgroup (C for BCSS, and D for OS) after propensity score matching (PSM). 
patients. We observed that early MBC had worse survival outcomes than propensity score-matched FBC.

In recent decades, we observed that early $\mathrm{MBC}$ incidence trends were stable. However, the incidence rates of early FBC changed significantly. The drastic drop between 2002 and 2003 seems to be temporally linked to the sharp decline in the use of postmenopausal hormone therapy (PHT) following the results of the Women's Health Initiative findings in 2002 on the adverse effects of combined PHT use, including increased risk of breast cancer (11). This phenomenon occurred in many countries worldwide with similar drops in PHT prescription and a concomitant decrease in breast cancer incidence $(12,13)$. There are other explanations for the decline in the incidence of FBC, such as a decrease in mammography screening $(14,15)$ and improvement lifestyle (16). However, these factors had only a modest effect on the incidence of breast cancer.

Additionally, in both $\mathrm{MBC}$ and $\mathrm{FBC}$, the unmarried patients in our study had worse BCSS and OS compared with married patients. Many studies reported that marital status had impacted cancer outcomes $(17,18)$. Previous reports illustrated that unmarried status was a significant predictor of the refusal of definitive treatment, early discontinuation of treatment, and non-adherence to adjuvant therapy in breast cancer patients $(19,20)$. There are some possible explanations for this finding. First, unmarried patients may lack cancer screening. As is known, early screening could lead to early cancer detection and effective treatment. Breast cancer screening is a well-known generally effective strategy to reduce breast-cancer related death and improve the quality of life. Hanske et al. (21) demonstrated that divorced/widowed/separated and never-married patients had a lower rate of breast cancer screening than the married group (74\% vs. $81 \%)$. As a result, unmarried patients may have more advanced tumor and worse prognosis. Second, unmarried patients may have fewer economic resources and social support than married patients. Spouses may encourage patients to seek medical attention for worrisome symptoms, to receive curative therapies, and to comply with prescribed treatments, all of which may result in better prognosis $(22,23)$. Interestingly, a study showed that male patients benefited more from marriage than did female patients. The reason for this may be considered that unmarried women receive greater social support from their relatives or friends than unmarried men (24). Third, unmarried patients may lack psychosocial support and may have more psychological stress. Accumulating evidence suggested that negative emotions may contribute to cancer progression and mortality $(25,26)$.
Taken together, these findings suggested that the impact that marriage, social, and psychological support could affect survival in breast cancer patients.

Previous reports suggested that MBC patients were presented with older age at diagnosis, more aggressive tumor biology, and more comorbidities, and therefore poorer survival compared with FBC patients $(5,27,28)$. In our study, we used the national cancer-registry database and confirmed the previous findings that MBC patients had worse survival outcomes in a real-world scenario. Since the sample size of MBC patients was significantly different from that of the FBC patients, we developed a 1:4 (MBC:FBC) propensity scorematched cohort, in which we still observed that MBC was associated with worse clinical outcomes. Several underlying reasons should be considered. First, $\mathrm{MBC}$ has more aggressive biological behavior than FBC. A study evaluating the 21gene recurrence score in $\mathrm{MBC}$ and $\mathrm{FBC}$ illustrated that high recurrence score $\geq 31$ was more common in male patients (12.4\% vs. $7.4 \%$ ) (6), suggesting that MBC tended to have a higher risk of tumor relapse. Second, the optimal strategies for $\mathrm{MBC}$ remain unclear and are extrapolated from knowledge about female counterparts. Due to majority of $\mathrm{MBC}$ were HR-positive tumor, endocrine therapy plays an important role in MBC. However, male patients' compliance is not as adequate as FBC patients. Previous studies found that MBC patients had lower adherence rates to receive adjuvant endocrine therapy because they were less likely to accept side effects than females, such as decreased libido, sleep disorder and thromboembolic events (29-31). As a result, lower compliance may lead to the poorer prognosis. Third, the mechanism of ER in MBC may be different from that in FBC. This is further supported by our findings that HR status was not prognostic in MBC patients, which was different from that of the FBC population. These results were consistent with findings from a study from Turkey (32). On the other hand, since the serum estrogen level of male adults is close to that of the postmenopausal women (33), the use of AI might have improved the clinical outcomes in ER-positive MBC patients. However, studies showed that AIs led to inferior survival compared to tamoxifen alone (34,35). A study by Pagani et al. speculated that the inferiority of AIs versus tamoxifen in men might be associated with gonadal physiology, and suggested that men should be used gonadal suppression when to use AIs (36). Therefore, further studies on the mechanism of endocrine therapy in MBC patients are essential. We should be cautious about using the management strategy from FBC in MBC patients and suggest that HR-positive MBCs should receive more aggressive treatments and more intensive 
surveillance plans comparison with FBC patients.

There are some limitations to our study. First, the SEER database does not provide information about comorbidities, which may affect the survival outcomes of male and female patients. Second, some therapy-associated data that greatly affect the prognosis of breast cancer, such as endocrine therapy and targeted therapy, are not available in the SEER database. Third, we do not have information regarding the family history of breast cancer and BRCA mutation data. BRCA germline mutations are strongly associated with breast cancer development, especially for MBC (37). Recently several studies demonstrated that other genetic mutations, such as CHEK2 and PALB2, were associated with increased risk of MBC (38), but data on these pathogenic variants are missing in SEER. Lastly, local recurrence is not recorded in the database, and only mortality data are available, which is limited to survival outcomes. However, despite these limitations, this is a large population-based cohort with long-term follow-up in male and female patients with stage I-II breast cancer. Similar to other studies using data from the SEER database, the cancer registry did not contain all of the relevant information, which may have led to an imbalance between male and female cohorts. We used the PSM analysis to attenuate the confounding variables to obtain reliable prognosis data.

\section{Conclusions}

In conclusion, the incidence trends for early MBC were stable, whereas the trends changed significantly for early FBC from 2000 to 2015. Early MBC had worse survival outcomes when compared with female counterparts. In the future, more basic, biological studies are needed to investigate the driven pathways that account for the aggressive behaviors and inferior clinical outcomes of MBC. Clinical trials might be necessary to confirm the validity of using strategies from FBC in MBC patients, and individualized treatment approaches should be developed.

\section{Acknowledgments}

Funding: This study was funded by the Yat-sen Scholarship of Young Scientist program of Sun Yat-sen Memorial Hospital, Sun Yat-sen University (Recipient: Kai Chen), and by the grants from the Sun Yat-sen Clinical Research Cultivating Program of Sun Yat-sen Memorial Hospital, Sun Yat-sen University (Recipient: Kai Chen), and the grants from the China Anti-aging Promoting Association (Recipient: Shunrong Li). This study was also supported by the National Natural Science Foundation of China (Grant\# 81672619, Recipient: Fengxi Su).

\section{Footnotes}

Conflicts of Interest: The authors have no conflicts of interest to declare.

Ethical Statement: The authors are accountable for all aspects of the work in ensuring that questions related to the accuracy or integrity of any part of the work are appropriately investigated and resolved.

\section{References}

1. Siegel RL, Miller KD, Jemal A. Cancer statistics, 2018. CA Cancer J Clin 2018;68:7-30.

2. Olver IN. Prevention of breast cancer. Med J Aust 2016;205:475-9.

3. Birnbaum JK, Duggan C, Anderson BO, et al. Early detection and treatment strategies for breast cancer in lowincome and upper middle-income countries: a modelling study. Lancet Glob Health 2018;6:e885-93.

4. Yap YS, Lu YS, Tamura K, et al. Insights Into Breast Cancer in the East vs the West: A Review. JAMA Oncol 2019. [Epub ahead of print].

5. Leone J, Zwenger AO, Leone BA, et al. Overall Survival of Men and Women With Breast Cancer According to Tumor Subtype: A Population-based Study. Am J Clin Oncol 2019;42:215-20.

6. Massarweh SA, Sledge GW, Miller DP, et al. Molecular Characterization and Mortality From Breast Cancer in Men. J Clin Oncol 2018;36:1396-404.

7. Cloyd JM, Hernandez-Boussard T, Wapnir IL. Outcomes of partial mastectomy in male breast cancer patients: analysis of SEER, 1983-2009. Ann Surg Oncol 2013;20:1545-50.

8. Cardoso F, Bartlett JMS, Slaets L, et al. Characterization of male breast cancer: results of the EORTC 10085/ TBCRC/BIG/NABCG International Male Breast Cancer Program. Ann Oncol 2018;29:405-17.

9. Kim HJ, Fay MP, Feuer EJ, et al. Permutation tests for joinpoint regression with applications to cancer rates. Stat Med 2000;19:335-51.

10. Lewis DR, Chen HS, Cockburn MG, et al. Early estimates of cancer incidence for 2015: Expanding to include estimates for white and black races. Cancer 2018;124:2192-204.

11. Rossouw JE, Anderson GL, Prentice RL, et al. Risks and benefits of estrogen plus progestin in healthy postmenopausal 
women: principal results from the Women's Health Initiative randomized controlled trial. JAMA 2002;288:321-33.

12. Katalinic A, Lemmer A, Zawinell A, et al. Trends in hormone therapy and breast cancer incidence - results from the German Network of Cancer Registries. Pathobiology 2009;76:90-7.

13. De P, Neutel CI, Olivotto I, et al. Breast cancer incidence and hormone replacement therapy in Canada. J Natl Cancer Inst 2010;102:1489-95.

14. Ravdin PM, Cronin KA, Howlader N, et al. The decrease in breast-cancer incidence in 2003 in the United States. N Engl J Med 2007;356:1670-4.

15. Kerlikowske K, Miglioretti DL, Buist DS, et al. Declines in invasive breast cancer and use of postmenopausal hormone therapy in a screening mammography population. J Natl Cancer Inst 2007;99:1335-9.

16. Verkooijen HM, Bouchardy C, Vinh-Hung V, et al. The incidence of breast cancer and changes in the use of hormone replacement therapy: a review of the evidence. Maturitas 2009;64:80-5.

17. Liu L, Chi YY, Wang AA, et al. Marital Status and Survival of Patients with Hormone Receptor-Positive Male Breast Cancer: A Surveillance, Epidemiology, and End Results (SEER) Population-Based Study. Med Sci Monit 2018;24:3425-41.

18. Song W, Tian C. The Effect of Marital Status on Survival of Patients with Gastrointestinal Stromal Tumors: A SEER Database Analysis. Gastroenterol Res Pract 2018;2018:5740823.

19. Aizer AA, Chen MH, Parekh A, et al. Refusal of curative radiation therapy and surgery among patients with cancer. Int J Radiat Oncol Biol Phys 2014;89:756-64.

20. Hershman DL, Kushi LH, Shao T, et al. Early discontinuation and nonadherence to adjuvant hormonal therapy in a cohort of 8,769 early-stage breast cancer patients. J Clin Oncol 2010;28:4120-8.

21. Hanske J, Meyer CP, Sammon JD, et al. The influence of marital status on the use of breast, cervical, and colorectal cancer screening. Prev Med 2016;89:140-5.

22. Lejeune C, Sassi F, Ellis L, et al. Socio-economic disparities in access to treatment and their impact on colorectal cancer survival. Int J Epidemiol 2010;39:710-7.

23. Kravdal H, Syse A. Changes over time in the effect of marital status on cancer survival. BMC Public Health 2011;11:804.

24. Aizer AA, Chen MH, McCarthy EP, et al. Marital status and survival in patients with cancer. J Clin Oncol 2013;31:3869-76.

25. Lehto US, Ojanen M, Dyba T, et al. Baseline psychosocial predictors of survival in localised breast cancer. $\mathrm{Br} \mathrm{J}$ Cancer 2006;94:1245-52.

26. Gidron Y, Ronson A. Psychosocial factors, biological mediators, and cancer prognosis: a new look at an old story. Curr Opin Oncol 2008;20:386-92.

27. Chen X, Liu X, Zhang L, et al. Poorer survival of male breast cancer compared with female breast cancer patients may be due to biological differences. Jpn J Clin Oncol 2013;43:954-63.

28. Gnerlich JL, Deshpande AD, Jeffe DB, et al. Poorer survival outcomes for male breast cancer compared with female breast cancer may be attributable to in-stage migration. Ann Surg Oncol 2011;18:1837-44.

29. Wibowo E, Pollock PA, Hollis N, et al. Tamoxifen in men: a review of adverse events. Andrology 2016;4:776-88.

30. Venigalla S, Carmona R, Guttmann DM, et al. Use and Effectiveness of Adjuvant Endocrine Therapy for Hormone Receptor-Positive Breast Cancer in Men. JAMA Oncol 2018;4:e181114.

31. Fentiman IS. The endocrinology of male breast cancer. Endocr Relat Cancer 2018;25:R365-73.

32. Arslan UY, Oksüzoğlu B, Ozdemir N, et al. Outcome of non-metastatic male breast cancer: 118 patients. Med Oncol 2012;29:554-60.

33. Dimitrov NV, Colucci P, Nagpal S. Some aspects of the endocrine profile and management of hormone-dependent male breast cancer. Oncologist 2007;12:798-807.

34. Eggemann H, Altmann U, Costa SD, et al. Survival benefit of tamoxifen and aromatase inhibitor in male and female breast cancer. J Cancer Res Clin Oncol 2018;144:337-41.

35. Eggemann H, Ignatov A, Smith BJ, et al. Adjuvant therapy with tamoxifen compared to aromatase inhibitors for 257 male breast cancer patients. Breast Cancer Res Treat 2013;137:465-70.

36. Pagani O, Regan MM, Walley BA, et al. Adjuvant exemestane with ovarian suppression in premenopausal breast cancer. N Engl J Med 2014;371:107-18.

37. Silvestri V, Barrowdale D, Mulligan AM, et al. Male breast cancer in BRCA1 and BRCA2 mutation carriers: pathology data from the Consortium of Investigators of Modifiers of BRCA1/2. Breast Cancer Res 2016;18:15.

38. Pritzlaff $M$, Summerour P, McFarland R, et al. Male breast cancer in a multi-gene panel testing cohort: insights and unexpected results. Breast Cancer Res Treat 2017;161:575-86.

Cite this article as: Wang Y, Chen K, Yang Y, Tan L, Chen L, Zhu L, Su F, Liu X, Li S. Incidence and survival outcomes of early male breast cancer: a population-based comparison with early female breast cancer. Ann Transl Med 2019;7(20):536. doi: 10.21037/atm.2019.10.04 